\title{
On the intertwinings of regular dilations
}

\author{
by Dumitru GaşPar and Nicolae Suciu (Timişoara)
}

Wtodzimierz Mlak in memoriam

\begin{abstract}
The aim of this paper is to find conditions that assure the existence of the commutant lifting theorem for commuting pairs of contractions (briefly, bicontractions) having (*-)regular dilations. It is known that in such generality, a commutant lifting theorem fails to be true. A positive answer is given for contractive intertwinings which doubly intertwine one of the components. We also show that it is possible to drop the doubly intertwining property for one of the components in some special cases, for instance for semi-subnormal bicontractions. As an application, a result regarding the existence of a unitary (isometric) dilation for three commuting contractions is obtained.
\end{abstract}

0. Introduction. It is well known that the theorem of B. Sz.-Nagy and C. Foias regarding the lifting of the commutant of a pair of contractions plays an important role in the applications of dilation theory in operator interpolation problems, optimization and control, in geology and geophysics. This is excellently illustrated in the book [5] of C. Foiaş and A. E. Frazho.

Lately, the dilation theory method was extended to the study of commuting multioperators by many authors (W. Mlak, M. Słociński, M. Kosiek, M. Ptak, E. Albrecht, V. Müller, R. E. Curto, F. H. Vasilescu, A. Octavio, B. Chevreau and others). In 1993, at the B. Sz.-Nagy Anniversary International Conference in Szeged, C. Foiaş raised the problem of obtaining a commutant lifting theorem for a pair of bicontractions having regular unitary dilations. In 1994, at the XV-th International Conference on Operator Theory in Timişoara, V. Müller proved that in such a generality, the commutant lifting theorem fails.

In the present work it is our aim to find conditions that assure the existence of such a lifting. In this frame a structure for regular (or $*$-regular) dilations is needed.

1991 Mathematics Subject Classification: Primary 47A20; Secondary 47A13.

Key words and phrases: commuting multioperator, *-regular dilation, contractive intertwining, (semi-)subnormal pair. 
1. Preliminaries. For a complex separable Hilbert space $\mathcal{H}, \mathcal{B}(\mathcal{H})$ means the $C^{*}$-algebra of all bounded linear operators on $\mathcal{H}$ (with Hilbert adjoint as involution). The elements of the (closed) unit ball in $\mathcal{B}(\mathcal{H})$ are called contractions on $\mathcal{H}$. An $n$-tuple of operators will be called a multioperator. If the members of the $n$-tuple commute, then we have a commuting multioperator. A commuting multioperator consisting of contractions will be called a multicontraction (bicontraction if $n=2$ ) on $\mathcal{H}$.

For a multicontraction $T:=\left(T_{1}, \ldots, T_{n}\right)$ we define $T^{*}:=\left(T_{1}^{*}, \ldots, T_{n}^{*}\right)$. We shall also use the multiindex notation

$$
T^{m}:=T_{1}^{m_{1}} \ldots T_{n}^{m_{n}}, \quad m=\left(m_{1}, \ldots, m_{n}\right) \in \mathbb{Z}_{+}^{n},
$$

where $\mathbb{Z}$ (resp. $\mathbb{Z}_{+}$) is the set of all (resp. positive) integers. A multicontraction $T$ on $\mathcal{H}$ will be briefly denoted by $[\mathcal{H}, T]$.

An isometric (resp. unitary) dilation of a multicontraction $[\mathcal{H}, T]$ is a multicontraction $[\mathcal{K}, U]$ consisting of isometric (resp. unitary) operators, such that $\mathcal{K}$ contains $\mathcal{H}$ as a closed subspace and

$$
T^{m}=P_{\mathcal{H}} U^{m} \mid \mathcal{H} \quad\left(m \in \mathbb{Z}_{+}^{n}\right),
$$

where $P_{\mathcal{H}}=P_{\mathcal{K}, \mathcal{H}}$ is the orthogonal projection of $\mathcal{K}$ on $\mathcal{H}$.

It is known (see [1]) that each bicontraction has an isometric (and unitary) dilation, and generally speaking, an $n$-tuple consisting of more than three commuting contractions has no isometric dilation (see [15]). An isometric, respectively unitary, dilation $[\mathcal{K}, U]$ of $[\mathcal{H}, T]$ is called minimal if

$$
\mathcal{K}=\bigvee_{m \in \mathbb{Z}_{+}^{n}} U^{m} \mathcal{H}
$$

or respectively,

$$
\mathcal{K}=\bigvee_{m \in \mathbb{Z}^{n}} U^{m} \mathcal{H}
$$

Let us first note that if $[\mathcal{K}, V]$ is an isometric minimal dilation of the multicontraction $[\mathcal{H}, T]$, then by $(2), \mathcal{H}$ is invariant with respect to $V^{*}$ and $V_{i}^{*} \mid \mathcal{H}=T_{i}^{*}(i=1, \ldots, n)$. Let us also mention that if $[\mathcal{K}, V]$ is an isometric minimal dilation of $[\mathcal{H}, T]$, and $[\widetilde{\mathcal{K}}, U]$ is the minimal unitary extension (see $[18])$ of $[\mathcal{K}, V]$, then it is the unitary minimal dilation of $[\mathcal{H}, T]$.

On the other hand, it is known that in case of a single contraction, the minimality condition (2) or $\left(2^{\prime}\right)$ implies that the isometric (resp. unitary) dilation is uniquely determined up to a unitary equivalence which fixes $\mathcal{H}$. But for $n>1$ this is not true ([1], [18]).

An isometric (resp. unitary) minimal dilation $[\mathcal{K}, U]$ of the multicontraction $[\mathcal{H}, T]$ is called regular (respectively $*$-regular) if it satisfies

$$
T^{* m_{-}} T^{m_{+}}=P_{\mathcal{H}} U^{* m_{-}} U^{m_{+}} \mid \mathcal{H} \quad\left(m \in \mathbb{Z}^{n}\right),
$$


or respectively,

$$
T^{m_{+}} T^{* m_{-}}=P_{\mathcal{H}} U^{* m_{-}} U^{m_{+}} \mid \mathcal{H} \quad\left(m \in \mathbb{Z}^{n}\right),
$$

where $m_{+}:=\left(m_{1}^{+}, \ldots, m_{n}^{+}\right), m_{-}=\left(m_{1}^{-}, \ldots, m_{n}^{-}\right)$and $m_{i}^{+}:=\max \left\{m_{i}, 0\right\}$, $m_{i}^{-}:=\max \left\{-m_{i}, 0\right\}$.

Regular dilations were studied in [3], [10], [18] and recently in [4] and [8]. Their existence is not assured for any multicontraction, not even for $n=2$. However, if such a dilation exists, then by the minimality condition $(2)$ or $\left(2^{\prime}\right)$ it is uniquely determined up to unitary equivalence (see [18]). It is easy to see from $(3)$ and $\left(3^{*}\right)$ that $[\mathcal{K}, U]$ is a regular (resp. *-regular) unitary dilation of $[\mathcal{H}, T]$ iff $\left[\mathcal{K}, U^{*}\right]$ is a $*$-regular (resp. regular) unitary dilation of $\left[\mathcal{H}, T^{*}\right]$. We also note that if $[\mathcal{K}, V]$ is a regular (resp. *-regular) isometric dilation of $[\mathcal{H}, T]$ then the minimal unitary extension $[\widetilde{\mathcal{K}}, U]$ of $[\mathcal{K}, V]$ is a regular (resp. *-regular) unitary dilation. On the other hand, if $[\mathcal{K}, U]$ is a regular unitary dilation, by putting $\mathcal{K}^{+}:=\bigvee_{m \in \mathbb{Z}_{+}^{n}} U^{m} \mathcal{H}$, $\mathcal{K}_{*}^{+}:=\bigvee_{m \in \mathbb{Z}_{+}^{n}} U^{* m} \mathcal{H}, \quad V_{* i}:=U_{i}^{*} \mid \mathcal{K}_{*}^{+}$and $V_{i}:=U_{i} \mid \mathcal{K}^{+}(i=1, \ldots, n)$, then $\left[\mathcal{K}^{+}, V\right]$ is a regular isometric dilation of $[\mathcal{H}, T]$, whereas $\left[\mathcal{K}_{*}^{+}, V_{*}\right]$ is a $*$-regular isometric dilation of $\left[\mathcal{H}, T^{*}\right]$. Let us also recall that a multicontraction $[\mathcal{H}, T]$ has a regular isometric dilation iff

$$
\Delta_{T}:=\sum_{|m| \leq n}(-1)^{|m|} T^{* m_{-}} T^{m_{+}} \geq 0
$$

where $|m|:=m_{1}+\ldots+m_{n}$ (see [18], [4]).

A multicontraction is called a polydisc isometry $([4])$ when $\Delta_{T}=0$. It is easily seen that if $T_{i}(i=1, \ldots, n)$ are isometries (i.e. $T$ is an $n$-toral isometry [2]), then $T$ is a polydisc isometry. Now if $I-\sum_{i=1}^{n} T_{i}^{*} T_{i} \geq 0$, then $\Delta_{T} \geq 0$. If $\sum_{i=1}^{n} T_{i}^{*} T_{i}=I$, then $[\mathcal{H}, T]$ is called a spherical isometry $([2])$. When $\left[\mathcal{H}, T^{*}\right]$ is a polydisc or a spherical isometry, we say that $[\mathcal{H}, T]$ is a polydisc or a spherical coisometry, respectively. If the multicontraction is doubly commuting, then obviously $\Delta_{T}=\left(I-T_{1}^{*} T_{1}\right) \ldots\left(I-T_{n}^{*} T_{n}\right) \geq 0$.

Furthermore, it is easy to verify

Proposition 0. For a multicontraction $[\mathcal{H}, T]$ the following statements are equivalent:

(i) $[\mathcal{H}, T]$ is doubly commuting;

(ii) $[\mathcal{H}, T]$ has a regular isometric dilation which is doubly commuting;

(iii) $[\mathcal{H}, T]$ has a regular unitary dilation $[\mathcal{K}, U]$ such that $\left[\mathcal{K}, U^{*}\right]$ is a regular unitary dilation for $\left[\mathcal{H}, T^{*}\right]$.

In particular, a multicontraction consisting of coisometries has a regular isometric (or unitary) dilation iff it is doubly commuting. 
Let us observe that Proposition 0(iii) means more than that $T$ has a regular and a *-regular dilation. For example, for a bicontraction $T=\left(T_{0}, T_{1}\right)$ with $\left\|T_{0}\right\|^{2}+\left\|T_{1}\right\|^{2} \leq 1$, we have $\Delta_{T} \geq 0$ and $\Delta_{T^{*}} \geq 0$ but it is possible that $T_{0} T_{1}^{*} \neq T_{1}^{*} T_{0}$.

Finally, also recall that an isometric pair $[\mathcal{H}, V]$ is called a shift $n$-tuple (see [7], [8]) or a multishift (see [4]) if there exists a wandering (closed) subspace $\mathcal{E}$ in $\mathcal{H}$ (i.e. $V^{m} \mathcal{E} \perp V^{p} \mathcal{E}, m \neq p, m, p \in \mathbb{Z}_{+}^{n}$ ) such that $\mathcal{H}=$ $\bigoplus_{m \in \mathbb{Z}_{+}^{n}} V^{m} \mathcal{E}$. For the sake of simplicity we shall work in the case $n=2$.

2. *-Regular isometric dilations. The isometric dilations consisting of doubly commuting isometries are in some sense connected with regular dilations. Precisely this is given in

Theorem 1. For a bicontraction $[\mathcal{H}, T]$ with $T=\left(T_{0}, T_{1}\right)$ the following assertions are equivalent:

(i) Thas a doubly commuting minimal isometric dilation;

(ii) $T$ has a minimal isometric dilation of the form $[\mathcal{M} \oplus \mathcal{G}, W \oplus V]$, where $W$ is a bishift on $\mathcal{M}$ and $V$ is a bidisc coisometry on $\mathcal{G}$;

(iii) If $\left[\mathcal{K}_{0}, S_{0}\right]$ is the minimal isometric dilation of $T_{0}$, then there exists a contraction $S_{1}$ on $\mathcal{K}_{0}$ which doubly commutes with $S_{0}$, such that $P_{\mathcal{H}} S_{1}=$ $T_{1} P_{\mathcal{H}}$

(iv) $T$ has a *-regular isometric (unitary) dilation.

Proof. (i) $\Rightarrow($ ii). Let $[\mathcal{K}, U]$ be a minimal isometric dilation of $T$ with $U_{0}, U_{1}$ doubly commuting isometries on $\mathcal{K}$. By the Wold decomposition ([17], [7]) we have $\mathcal{K}=\mathcal{K}_{u} \oplus \mathcal{K}_{s} \oplus \mathcal{K}_{s 0} \oplus \mathcal{K}_{s 1}$, so that $U_{0}$ and $U_{1}$ reduce each subspace and $U_{0}, U_{1}$ are unitary on $\mathcal{K}_{u}, U$ is a shift pair on $\mathcal{K}_{s}$ and $U_{i}$ is unitary (resp. a shift) on $\mathcal{K}_{s 1-i}\left(\right.$ resp. $\left.\mathcal{K}_{s i}\right), i=0,1$. Put $\mathcal{G}=\mathcal{K}_{u} \oplus \mathcal{K}_{s 0} \oplus \mathcal{K}_{s 1}$, $V_{i}=V_{i}^{i} \oplus V_{i}^{1-i}$ with $V_{i}^{i}=U_{i}\left|\mathcal{K}_{u} \oplus \mathcal{K}_{s 0}, V_{i}^{1-i}=U_{i}\right| \mathcal{K}_{s 1}(i=0,1)$ and $V=\left(V_{0}, V_{1}\right), W^{\prime}=\left(V_{0}^{0}, V_{1}^{1}\right), W^{\prime \prime}=\left(V_{0}^{1}, V_{1}^{0}\right)$. Because $V_{1}^{1}$ is unitary, $W^{\prime *}$ is a bidisc isometry on $\mathcal{K}_{u} \oplus \mathcal{K}_{s 0}$, and since $V_{0}^{1}$ is unitary, $W^{\prime \prime *}$ is a bidisc isometry on $\mathcal{K}_{s 1}$. Then

$$
\Delta_{V^{*}}=\Delta_{W^{\prime *}}+\Delta_{W^{\prime *}}=0,
$$

so $V^{*}$ is a bidisc isometry on $\mathcal{G}$. Therefore since $W:=\left(U_{0}\left|\mathcal{K}_{s}, U_{1}\right| \mathcal{K}_{s}\right)$ is a bishift on $\mathcal{M}=\mathcal{K}_{s}$ and $\mathcal{K}=\mathcal{M} \oplus \mathcal{G}, W \oplus V=U$, we see that the dilation $[\mathcal{K}, U]$ of $T$ has the form described in (ii)

(ii) $\Rightarrow$ (iii). Let $[\mathcal{M} \oplus \mathcal{G}, W \oplus V]$ be as in (ii). Since $W$ is a bishift on $\mathcal{M}$, the isometries $W_{0}$ and $W_{1}$ doubly commute on $\mathcal{M}$ ([16]). Also the isometries $V_{0}$ and $V_{1}$ doubly commute on $\mathcal{G}$, because $V$ has a $*$-regular dilation. Therefore the isometries $U_{0}=W_{0} \oplus V_{0}$ and $U_{1}=W_{1} \oplus V_{1}$ doubly commute on $\mathcal{M} \oplus \mathcal{G}$. 
Put

$$
\mathcal{K}_{0}=\bigvee_{m \in \mathbb{Z}_{+}} W_{0}^{m} \mathcal{H}, \quad S_{0}=U_{0}\left|\mathcal{K}_{0}, \quad S_{1}=P_{\mathcal{K}_{0}} U_{1}\right| \mathcal{K}_{0}
$$

Then $\left[\mathcal{K}_{0}, S_{0}\right]$ is the minimal isometric dilation of $T_{0}, S_{0} S_{1}=S_{1} S_{0}$ and $P_{\mathcal{H}} S_{1}=T_{1} P_{\mathcal{H}}$. Since $U_{0}^{*}\left|\mathcal{H}=T_{0}^{*}=S_{0}^{*}\right| \mathcal{H}$, we also have $U_{0}^{*} \mid \mathcal{K}_{0}=S_{0}^{*}$. Furthermore, for $k=\sum_{p \in \mathbb{Z}_{+}} S_{0}^{p} h_{p} \in \mathcal{K}$ with the sequence $\left\{h_{p}\right\} \subset \mathcal{H}$ with finite support, we obtain

$$
\begin{aligned}
S_{1} S_{0}^{*} k & =S_{1} S_{0}^{*} h_{0}+\sum_{p \geq 1} S_{1} S_{0}^{p-1} h_{p} \\
& =P_{\mathcal{K}_{0}} U_{1} U_{0}^{*} h_{0}+\sum_{p \geq 1} S_{0}^{p-1} S_{1} h_{p} \\
& =P_{\mathcal{K}_{0}} U_{0}^{*} U_{1} h_{0}+\sum_{p \geq 1} S_{0}^{*} S_{0}^{p} S_{1} h_{p} \\
& =S_{0}^{*} S_{1} h_{0}+S_{0}^{*} \sum_{p \geq 1} S_{1} S_{0}^{p} h_{p}=S_{0}^{*} S_{1} k,
\end{aligned}
$$

where we have used the fact that $P_{\mathcal{K}_{0}} U_{0}^{*} U_{1} \mid \mathcal{K}_{0}=S_{0}^{*} S_{1}$. Consequently, $S_{0}$ and $S_{1}$ doubly commute on $\mathcal{K}_{0}$.

(iii) $\Rightarrow$ (iv). If $\mathcal{K}_{0}, S_{0}$ and $S_{1}$ are as in (iii), then $S_{i}^{*} \mid \mathcal{H}=T_{i}^{*}(i=0,1)$ and since $S_{0}$ and $S_{1}$ doubly commute, we have

$$
\begin{aligned}
\Delta_{T^{*}} & =I-T_{0} T_{0}^{*}-T_{1} T_{1}^{*}+T_{0} T_{1} T_{0}^{*} T_{1}^{*} \\
& =P_{\mathcal{H}}\left(I-S_{0} S_{0}^{*}-S_{1} S_{1}^{*}+S_{0} S_{1} S_{0}^{*} S_{1}^{*}\right) \mid \mathcal{H} \\
& =P_{\mathcal{H}}\left(I-S_{0} S_{0}^{*}\right)\left(I-S_{1} S_{1}^{*}\right) \mid \mathcal{H} \geq 0 .
\end{aligned}
$$

Consequently, $T$ has a $*$-regular isometric (or unitary) dilation.

(iv) $\Rightarrow$ (i). Suppose $\Delta_{T^{*}} \geq 0$. Denote by $\mathcal{M}=H^{2}\left(\mathbf{T}^{2}, \mathcal{H}\right)$ and $Z=$ $\left(Z_{0}, Z_{1}\right)$ the shift pair (that is, $Z_{0}$ and $Z_{1}$ are the operators of multiplication with the coordinate functions) on $\mathcal{M}$. Using Theorem 3.15 of [4], there are a Hilbert space $\mathcal{H}_{1}$, a bicontraction $N=\left(N_{0}, N_{1}\right)$ on $\mathcal{H}_{1}$ with $N_{0}$ and $N_{1}$ normal operators and with $N^{*}$ a bidisc isometry, and an isometry $A$ of $\mathcal{H}$ in $\mathcal{M} \oplus \mathcal{H}_{1}$ such that $A \mathcal{H}$ is invariant for $\left(Z_{i} \oplus N_{i}\right)^{*}$ and $\left(Z_{i} \oplus N_{i}\right)^{*} A=A T_{i}^{*}$, $i=0,1$. Then it results that

$$
T_{0}^{p} T_{1}^{q}=A^{*}\left(Z_{0} \oplus N_{0}\right)^{p}\left(Z_{1} \oplus N_{1}\right)^{q} A \quad\left(p, q \in \mathbb{Z}_{+}\right) .
$$

Let now $\left[\mathcal{K}_{1},\left(M_{0}, M_{1}\right)\right]$ be a minimal isometric dilation of $N$ with $M_{0}$ and $M_{1}$ doubly commuting isometries on $\mathcal{K}_{1}$. Put

$$
\mathcal{K}=\mathcal{M} \oplus \mathcal{K}_{1}, \quad U_{i}=Z_{i} \oplus M_{i} \quad(i=0,1) .
$$

Then $U_{0}$ and $U_{1}$ are doubly commuting isometries on $\mathcal{K}$. Denoting by $J$ the embedding of $\mathcal{M} \oplus \mathcal{H}_{1}$ in $\mathcal{K}$, we find that $J A$ is an isometry of $\mathcal{H}$ into $\mathcal{K}$. 
For $m=(p, q) \in \mathbb{Z}_{+}^{2}$ and $h \in H$ we obtain

$$
\begin{aligned}
(J A)^{*} U^{m} J A h & =(J A)^{*} U_{0}^{p} U_{1}^{q}\left(P_{\mathcal{H}_{0}} A h \oplus P_{\mathcal{H}_{1}} A h\right) \\
& =(J A)^{*}\left(Z^{m} P_{\mathcal{H}_{0}} A h \oplus M^{m} P_{\mathcal{H}_{1}} A h\right) \\
& =A^{*}\left(P_{\mathcal{H}_{0}} Z^{m} P_{\mathcal{H}_{0}} A h \oplus P_{\mathcal{H}_{1}} M^{m} P_{\mathcal{H}_{1}} A h\right) \\
& =A^{*}\left(Z^{m} P_{\mathcal{H}_{0}} A h \oplus N^{m} P_{\mathcal{H}_{1}} A h\right) \\
& =A^{*}\left(Z_{0} \oplus N_{0}\right)^{p}\left(Z_{1} \oplus N_{1}\right)^{q} A h=T^{m} h .
\end{aligned}
$$

Let us observe that the subspace $A \mathcal{H}$ is invariant for $U_{i}^{*}(i=0,1)$, because for $h \in \mathcal{H}$ we have

$$
\begin{aligned}
U_{i}^{*} A h & =U_{i}^{*}\left(P_{\mathcal{H}_{0}} A h \oplus P_{\mathcal{H}_{1}} A h\right)=Z_{i}^{*} P_{\mathcal{H}_{0}} A h \oplus M_{i}^{*} P_{\mathcal{H}_{1}} A h \\
& =Z_{i}^{*} P_{\mathcal{H}_{0}} A h \oplus N_{i}^{*} P_{\mathcal{H}_{1}} A h=\left(Z_{i} \oplus N_{i}\right)^{*} A h=A T_{i}^{*} h .
\end{aligned}
$$

Now define

$$
\mathcal{K}_{+}=\bigvee_{m \in \mathbb{Z}_{+}^{2}} U^{m} A h, \quad V_{i}=U_{i} \mid \mathcal{K}_{+} \quad(i=0,1)
$$

and $B=J_{+} A$, where $J_{+}$is the embedding of $\mathcal{M} \oplus \mathcal{H}_{1}$ in $\mathcal{K}_{+}$. Then $B$ is an isometry of $\mathcal{H}$ into $\mathcal{K}_{+}$and we have

$$
T^{m}=B^{*} V^{m} B \quad\left(m \in \mathbb{Z}_{+}^{2}\right) .
$$

Identifying $\mathcal{H}$ with $B \mathcal{H}$ in $\mathcal{K}_{+}$, we deduce that $\left[\mathcal{K}_{+}, V\right]$ is a minimal isometric dilation of $T$. It remains to prove that $V_{0} V_{1}^{*}=V_{1}^{*} V_{0}$. First, since $U_{0}$ and $U_{1}$ doubly commute on $\mathcal{K}$, it results that $\mathcal{K}_{+}$is invariant for $U_{i}^{*}, i=0,1$. Indeed, for $k=\sum_{m \in \mathbb{Z}_{+}^{2}} U^{m} A h_{m}$ with the sequence $\left\{h_{m}\right\} \subset \mathcal{H}$ with finite support, we obtain

$$
\begin{aligned}
U_{0}^{*} k & =\sum_{q \geq 0} U_{0}^{*} U_{1}^{q} A h_{0 q}+\sum_{\substack{p \geq 1 \\
q \geq 0}} U_{0}^{*} U_{0}^{p} U_{1}^{q} A h_{p q} \\
& =\sum_{q \geq 0} U_{1}^{q} U_{0}^{*} A h_{0 q}+\sum_{\substack{p \geq 1 \\
q \geq 0}} U_{0}^{p-1} U_{1}^{q} A h_{p q} \\
& =\sum_{q \geq 0} U_{1}^{q} A T_{0}^{*} h_{0 q}+\sum_{\substack{p \geq 1 \\
q \geq 0}} U_{0}^{p-1} U_{1}^{q} A h_{p q} .
\end{aligned}
$$

Therefore $U_{0}^{*} \mathcal{K}_{+} \subset \mathcal{K}_{+}$and analogously $U_{1}^{*} \mathcal{K}_{+} \subset \mathcal{K}_{+}$. Then $V_{i}^{*}=U_{i}^{*} \mid \mathcal{K}_{+}$ $(i=0,1)$, and consequently, $V_{0} V_{1}^{*}=V_{1}^{*} V_{0}$. Hence $\left[\mathcal{K}_{+},\left(V_{0}, V_{1}\right)\right]$ is a doubly commuting minimal isometric dilation of $T$.

COROLlary 2. A bicontraction $T$ on $\mathcal{H}$ has a regular isometric (unitary) dilation if and only if $T$ has a doubly commuting minimal coisometric extension. 
Proof. If $\Delta_{T} \geq 0$, then $T^{*}$ has a doubly commuting minimal isometric dilation $\left[\mathcal{K},\left(W_{0}, W_{1}\right)\right]$. Hence $\left(W_{0}^{*}, W_{1}^{*}\right)$ is a minimal coisometric extension of $T$ and $W_{0}^{*}, W_{1}^{*}$ doubly commute on $\mathcal{K}$. The converse is obvious.

Remark. If $\mathcal{K}=\mathcal{K}_{u}^{i} \oplus \mathcal{K}_{s}^{i}$ is the Wold decomposition of $\mathcal{K}$ relative to $W_{i}$ (in the previous proof), then $W_{1-i}$ reduces $\mathcal{K}_{u}^{i}$ and $\mathcal{K}_{s}^{i}, i=0,1$. Thus, the matrix of $W_{1-i}^{*}$ relative to the decomposition $\mathcal{K}=\mathcal{K}_{u}^{i} \oplus \mathcal{K}_{s}^{i}$ has diagonal form for $i=0,1$, that is, $T$ is diagonally extendable (see [11]).

Now we can give the following characterization of the double commutativity of an isometric dilation of $[\mathcal{H}, T]$.

Proposition 3. Suppose $T=\left(T_{0}, T_{1}\right)$ is a bicontraction on $\mathcal{H}$ and $\left[\mathcal{K},\left(U_{0}, U_{1}\right)\right]$ a minimal isometric dilation of $T$. Then the isometries $U_{0}$ and $U_{1}$ doubly commute on $\mathcal{K}$ iff $[\mathcal{K}, U]$ is a $*$-regular isometric dilation of $[\mathcal{H}, T]$. In particular, the doubly commuting minimal isometric dilation of $T$ (if it exists) is unique up to unitary equivalence.

Proof. It is not difficult to see that the condition $\left(3^{*}\right)$ is equivalent to

$$
T_{i}^{p} T_{1-i}^{* q}=P_{\mathcal{H}} U_{1-i}^{* q} U_{i}^{p} \mid \mathcal{H} \quad\left(p, q \in \mathbb{Z}_{+} ; i=0,1\right) .
$$

Suppose that the dilation $U=\left(U_{0}, U_{1}\right)$ satisfies $(4)$ and let $\left[\widetilde{\mathcal{K}},\left(\widetilde{U_{0}}, \widetilde{U_{1}}\right)\right]$ be the minimal unitary extension of $U$. Then for $p, q \in \mathbb{Z}_{+}$and $i=0,1$ we have

$$
T_{i}^{p} T_{1-i}^{* q}=P_{\mathcal{H}} U_{1-i}^{* q} U_{i}^{p}\left|\mathcal{H}=P_{\mathcal{H}} \widetilde{U}_{i}^{p} \widetilde{U}_{1-i}^{* q}\right| \mathcal{H}
$$

and we deduce that $\left[\widetilde{\mathcal{K}},\left(\widetilde{U}_{0}^{*}, \mathcal{U}_{1}^{*}\right)\right]$ is a regular minimal unitary dilation for $T^{*}$. By Theorem 1, $T$ has a doubly commuting minimal isometric dilation $\left[\mathcal{M},\left(V_{0}, V_{1}\right)\right]$. Obviously, $V_{i}$ satisfies (4) (in place of $U_{i}$ ) and consequently

$$
P_{\mathcal{H}} U_{i}^{* q} U_{1-i}^{p}\left|\mathcal{H}=P_{\mathcal{H}} V_{i}^{* q} V_{1-i}^{p}\right| \mathcal{H} \quad\left(p, q \in \mathbb{Z}_{+} ; i=0,1\right) .
$$

Let us prove that the dilations $U=\left(U_{0}, U_{1}\right)$ and $V=\left(V_{0}, V_{1}\right)$ are unitarily equivalent. Let $\left\{h_{n}\right\}_{n \in \mathbb{Z}_{+}^{2}} \subset \mathcal{H}$ be a sequence with finite support. Since $U$ and $V$ are dilations of $T$ and satisfy $(*)$, by defining $m:=(i, j) \in \mathbb{Z}_{+}^{2}$ and $n:=(p, q) \in \mathbb{Z}_{+}^{2}$ we obtain

$$
\begin{aligned}
& \left\|\sum_{n \in \mathbb{Z}_{+}^{2}} U^{n} h_{n}\right\|^{2}=\sum_{m, n \in \mathbb{Z}_{+}^{2}}\left(U^{n} h_{n}, U^{m} h_{m}\right) \\
& =\sum_{j<q}\left(U_{0}^{* i} U_{0}^{p} U_{1}^{q-j} h_{n}, h_{m}\right)+\sum_{j \geq q}\left(U_{0}^{* i} U_{1}^{*(j-q)} U_{0}^{p} h_{n}, h_{m}\right) \\
& =\sum_{\substack{i<p \\
j<q}}\left(U_{0}^{p-i} U_{1}^{q-j} h_{n}, h_{m}\right)+\sum_{\substack{i \geq p \\
j<q}}\left(U_{0}^{*(i-p)} U_{1}^{q-j} h_{n}, h_{m}\right)
\end{aligned}
$$




$$
\begin{aligned}
& +\sum_{\substack{i<p \\
j \geq q}}\left(U_{1}^{*(j-q)} U_{0}^{p-i} h_{n}, h_{m}\right)+\sum_{\substack{i \geq p \\
j \geq q}}\left(U_{0}^{*(i-p)} U_{1}^{*(j-q)} h_{n}, h_{m}\right) \\
= & \sum_{\substack{i<p \\
j<q}}\left(V_{0}^{p-i} V_{1}^{q-j} h_{n}, h_{m}\right)+\sum_{\substack{i \geq p \\
j<q}}\left(V_{0}^{*(i-p)} V_{1}^{q-j} h_{n}, h_{m}\right) \\
& +\sum_{\substack{i<p \\
j \geq q}}\left(V_{1}^{*(j-q)} V_{0}^{p-i} h_{n}, h_{m}\right)+\sum_{\substack{i \geq p \\
j \geq q}}\left(V_{0}^{*(i-p)} V_{1}^{*(j-q)} h_{n}, h_{m}\right) \\
= & \sum_{m, n \in \mathbb{Z}_{+}^{2}}\left(V^{* m} V^{n} h_{n}, h_{m}\right)=\left\|\sum_{\substack{n \in \mathbb{Z}_{+}^{2}\\
}} V^{n} h_{n}\right\|^{2} .
\end{aligned}
$$

Using the minimality conditions of the spaces $\mathcal{K}$ and $\mathcal{M}$ and the norm equalities above, we deduce that there exists a unitary operator $W$ from $\mathcal{K}$ to $\mathcal{M}$ satisfying

$$
W \sum_{n \in \mathbb{Z}_{+}^{2}} U^{n} h_{n}=\sum_{n \in \mathbb{Z}_{+}^{2}} V^{n} h_{n}
$$

for $\left\{h_{n}\right\} \subset \mathcal{H}$ with finite support. Consequently, $W \mid \mathcal{H}=I$ and $W U_{i}=V_{i} W$, $i=0,1$, and in particular, it results that $U_{0}$ and $U_{1}$ doubly commute on $\mathcal{K}$. Since the other assertions were also implicitly proved, the proof is finished.

Now, having in mind the condition (iii) of Theorem 1, we obtain

Corollary 4. Let $T=\left(T_{0}, T_{1}\right)$ be a bicontraction on $\mathcal{H}$ and $\left[\mathcal{K}_{0}, S_{0}\right]$ (respectively $\left.\left[\mathcal{K}_{* 0}, S_{* 0}\right]\right)$ the minimal isometric dilation of $T_{0}\left(\right.$ resp. $\left.T_{0}^{*}\right)$. Then $T$ has a *-regular (resp. regular) isometric dilation if and only if $T_{1}^{*}$ (resp. $\left.T_{1}\right)$ has a contractive extension on $\mathcal{K}_{0}\left(\right.$ resp. $\left.\mathcal{K}_{* 0}\right)$ which doubly commutes with $S_{0}\left(\right.$ resp. $\left.S_{* 0}\right)$.

It is obvious (by the proof of Proposition 3 ) that if $[\mathcal{K}, V]$ is a $*$-regular (resp. regular) isometric dilation of $[\mathcal{H}, T]$ and if $[\widetilde{\mathcal{K}}, U]$ is the minimal unitary extension of $V$ then the regular (resp. *-regular) isometric dilation of $T^{*}$ is $\left[\mathcal{K}_{*},\left(V_{* 0}, V_{* 1}\right)\right]$, where

$$
\mathcal{K}_{*}=\bigvee_{m, n \in \mathbb{Z}_{+}} U_{0}^{* m} U_{1}^{* n} \mathcal{H}, \quad V_{* i}=U_{i}^{*} \mid \mathcal{K}_{*} \quad(i=0,1) .
$$

Furthermore, with the notations of Theorem 1(iii), the *-regular isometric dilation $[\mathcal{K}, V]$ of $T$ is the regular and $*$-regular isometric dilation of the doubly commuting bicontraction $S=\left(S_{0}, S_{1}\right)$ (see Proposition 0), and in fact, $\left[\mathcal{K}, V_{1}\right]$ is the minimal isometric dilation of $S_{1}$.

3. Intertwinings of regular dilations. Let $\mathcal{H}$ and $\mathcal{H}^{\prime}$ be two Hilbert spaces and $T=\left(T_{0}, T_{1}\right)$ and $T^{\prime}=\left(T_{0}^{\prime}, T_{1}^{\prime}\right)$ two bicontractions on $\mathcal{H}$ and $\mathcal{H}^{\prime}$ respectively. A bounded linear operator $A: \mathcal{H} \rightarrow \mathcal{H}^{\prime}$ intertwines $T$ and 
$T^{\prime}$ if $A T_{i}=T_{i}^{\prime} A, i=0,1$. The operator $A$ doubly intertwines $T$ and $T^{\prime}$ if $A T_{i}=T_{i}^{\prime} A$ and $A T_{i}^{*}=T_{i}^{\prime *} A, i=0,1$.

V. Müller has shown in [14] that if $A$ intertwines two bicontractions which have regular dilations, then in general, $A$ cannot be "lifted" in the sense of [5], [18] to an operator which intertwines these dilations. In order to give conditions under which this is possible, we will first prove

TheOREM 5. Let $[\mathcal{H}, T]$ and $\left[\mathcal{H}^{\prime}, T^{\prime}\right]$ be two bicontractions having *regular isometric dilations $[\mathcal{K}, U]$ and $\left[\mathcal{K}^{\prime}, U^{\prime}\right]$ respectively. Let $A$ be a contraction from $\mathcal{H}$ in $\mathcal{H}^{\prime}$ such that $A T_{i}=T_{i}^{\prime} A(i=0,1)$ and $A T_{0}^{*}=T_{0}^{\prime *} A$. Then there is a contraction $B$ from $\mathcal{K}$ in $\mathcal{K}^{\prime}$ with $B U_{i}=U_{i}^{\prime} B(i=0,1)$, $B U_{0}^{*}=U_{0}^{\prime *}$ and $P_{\mathcal{H}^{\prime}} B=A P_{\mathcal{H}}$.

Proof. Let $A: \mathcal{H} \rightarrow \mathcal{H}^{\prime}$ be a contraction which satisfies $A T_{i}=T_{i}^{\prime} A$ $(i=0,1)$ and $A T_{0}^{*}=T_{0}^{\prime *} A$. Let $\left[\mathcal{K}_{0}, S_{0}\right]$ and $\left[\mathcal{K}_{0}^{\prime}, S_{0}^{\prime}\right]$ be the minimal isometric dilations of $T_{0}$ and $T_{0}^{\prime}$ respectively. By Theorem 1(iii) there are contractions $S_{1}$ on $\mathcal{K}_{0}$ and $S_{1}^{\prime}$ on $\mathcal{K}_{0}^{\prime}$ such that $S_{1}$ doubly commutes with $S_{0}$ and $P_{\mathcal{H}} S_{1}=T_{1} P_{\mathcal{H}}$, while $S_{1}^{\prime}$ doubly commutes with $S_{0}^{\prime}$ and $P_{\mathcal{H}^{\prime}} S_{1}^{\prime}=T_{1}^{\prime} P_{\mathcal{H}^{\prime}}$. Since

$$
\mathcal{K}_{0}=\mathcal{H} \oplus \bigoplus_{p \in \mathbb{Z}_{+}} S_{0}^{p} \overline{\left(S_{0}-T_{0}\right) \mathcal{H}}, \quad \mathcal{K}_{0}^{\prime}=\mathcal{H}^{\prime} \oplus \bigoplus_{p \in \mathbb{Z}_{+}} S_{0}^{\prime p} \overline{\left(S_{0}^{\prime}-T_{0}^{\prime}\right) \mathcal{H}^{\prime}}
$$

(see [5], [18]), and $A$ doubly intertwines $T_{0}$ and $T_{0}^{\prime}$, we can define a contraction $A_{0}: \mathcal{K}_{0} \rightarrow \mathcal{K}_{0}^{\prime}$ by setting

$$
A_{0} k_{0}:=A h+\sum_{p \geq 0} S_{0}^{\prime p}\left(S_{0}^{\prime}-T_{0}^{\prime}\right) A h_{p}
$$

for $k_{0}=h+\sum_{p \in \mathbb{Z}_{+}} S_{0}^{p}\left(S_{0}-T_{0}\right) h_{p}$, where $h, h_{p} \in \mathcal{H}$. We have $A_{0} \mid \mathcal{H}=A$, and for $k_{0} \in \mathcal{K}_{0}$ as above,

$$
\begin{aligned}
A_{0} S_{0} k_{0} & =A_{0}\left[T_{0} h+\left(S_{0}-T_{0}\right) h+\sum_{p \geq 0} S_{0}^{p+1}\left(S_{0}-T_{0}\right) h_{p}\right] \\
& =A T_{0} h+\left(S_{0}^{\prime}-T_{0}^{\prime}\right) A h+\sum_{p \geq 0} S_{0}^{\prime p+1}\left(S_{0}^{\prime}-T_{0}^{\prime}\right) A h_{p} \\
& =S_{0}^{\prime}\left[A h+\sum_{p \geq 0} S_{0}^{\prime p}\left(S_{0}^{\prime}-T_{0}^{\prime}\right) A h_{p}\right]=S_{0}^{\prime} A_{0} k_{0} .
\end{aligned}
$$

Therefore $A_{0} S_{0}=S_{0}^{\prime} A_{0}$. Also, $A_{0} S_{0}^{*}=S_{0}^{\prime *} A_{0}$ and $A_{0}^{*} \mid \mathcal{H}^{\prime}=A^{*}$, because for $k=\sum_{p \geq 0} S_{0}^{p} h_{p}$ (finite sum) with $h_{p} \in \mathcal{H}$, we have

$$
\begin{aligned}
A_{0} S_{0}^{*} k & =A T_{0}^{*} h_{0}+\sum_{p \geq 1} A_{0} S_{0}^{p-1} h_{p}=T_{0}^{\prime *} A h_{0}+\sum_{p \geq 1} S_{0}^{\prime p-1} A h_{p} \\
& =S_{0}^{* *} A h_{0}+S_{0}^{\prime *} \sum_{p \geq 1} S_{0}^{\prime p} A h_{p}=S_{0}^{\prime *} \sum_{p \geq 0} S_{0}^{\prime p} A h_{p}=S_{0}^{\prime *} A_{0} k
\end{aligned}
$$


and for $h^{\prime} \in \mathcal{H}^{\prime}$,

$$
\begin{aligned}
\left(A_{0}^{*} h^{\prime}, k\right) & =\sum_{p}\left(h^{\prime}, S_{0}^{\prime p} A h_{p}\right)=\sum_{p}\left(T_{0}^{\prime * p} h^{\prime}, A h_{p}\right)=\sum_{p}\left(A^{*} T_{0}^{\prime * p} h^{\prime}, h_{p}\right) \\
& =\sum_{p}\left(T_{0}^{* p} A^{*} h^{\prime}, h_{p}\right)=\sum_{p}\left(A^{*} h^{\prime}, S_{0}^{p} h_{p}\right)=\left(A^{*} h^{\prime}, k\right) .
\end{aligned}
$$

Next, we also have $A_{0} S_{1}=S_{1}^{\prime} A_{0}$, because for $\left\{h_{p}^{\prime}\right\}_{p \geq 0} \subset \mathcal{H}^{\prime}$ with finite support,

$$
\begin{aligned}
A_{0}^{*} S_{1}^{\prime *} \sum_{p} S_{0}^{\prime p} h_{p}^{\prime} & =\sum_{p} A_{0}^{*} S_{0}^{\prime p} S_{1}^{\prime *} h_{p}^{\prime}=\sum_{p} S_{0}^{p} A_{0}^{*} T_{1}^{\prime *} h_{p}^{\prime}=\sum_{p} S_{0}^{p} A^{*} T_{1}^{\prime *} h_{p}^{\prime} \\
& =\sum_{p} S_{0}^{p} T_{1}^{*} A^{*} h_{p}^{\prime}=\sum_{p} S_{0}^{p} S_{1}^{*} A_{0}^{*} h_{p}^{\prime}=S_{1}^{*} A_{0}^{*} \sum_{p} S_{0}^{\prime p} h_{p}^{\prime},
\end{aligned}
$$

and consequently, $A_{0}^{*} S_{1}^{*}=S_{1}^{*} A_{0}^{*}$, whence $A_{0} S_{1}=S_{1}^{\prime} A_{0}$. We conclude that $A$ intertwines $S_{1}$ and $S_{1}^{\prime}$ and doubly intertwines $S_{0}$ and $S_{0}^{\prime}$, and $A_{0}$ is an extension for $A$, while $A_{0}^{*}$ is an extension for $A^{*}$. Hence $P_{\mathcal{H}^{\prime}} A_{0}=A P_{\mathcal{H}}$.

Now let $\left[\mathcal{K}, U_{1}\right]$, $\left[\mathcal{K}^{\prime}, U_{1}^{\prime}\right]$ be the minimal isometric dilations of $S_{1}, S_{1}^{\prime}$ respectively, and let $U_{0}, U_{0}^{\prime}$ be the $*$-extensions of $S_{0}\left(\right.$ on $\mathcal{K}$ ) and of $S_{0}^{\prime}$ (on $\mathcal{K}^{\prime}$ ), respectively, such that $U_{0}$ doubly commutes with $U_{1}$ and $U_{0}^{\prime}$ doubly commutes with $U_{1}^{\prime}$. Using the sequences of $n$-step dilations for $S_{1}$ and $S_{1}^{\prime}$ and the corresponding $n$-step intertwining liftings of $A$, we can define a contraction $B: \mathcal{K} \rightarrow \mathcal{K}^{\prime}$ by

$$
B k=\lim _{n} A_{n} P_{\mathcal{K}_{n}} k \quad(k \in \mathcal{K}),
$$

where $\left\{\mathcal{K}_{n}\right\}$ and $\left\{A_{n}\right\}$ are inductively defined with $\mathcal{K}_{1}=\mathcal{K}_{0} \oplus \mathcal{D}_{S_{1}}$ and $A_{1}: \mathcal{K}_{0} \oplus \mathcal{D}_{S_{1}} \rightarrow \mathcal{K}_{0}^{\prime} \oplus \mathcal{D}_{S_{1}^{\prime}}$ of the form

$$
A_{1}=\left(\begin{array}{cc}
A_{0} & 0 \\
X_{1} D_{A_{0}} & Y_{1}
\end{array}\right),
$$

$\mathcal{D}_{C}$ being the defect space of the operator $C$. Here the operator $\left(X_{1}, Y_{1}\right)$ : $\mathcal{D}_{A_{0}} \oplus \mathcal{D}_{S_{1}} \rightarrow \mathcal{D}_{S_{1}^{\prime}}$ is $\left(X_{1}, Y_{1}\right)=\Gamma_{0} P_{0}$, where $P_{0}$ is the orthogonal projection of $\mathcal{D}_{A_{0}} \oplus \mathcal{D}_{S_{1}}$ on the subspace $\left\{D_{A_{0}} S_{1} k \oplus D_{S_{1}} k: k \in \mathcal{K}_{0}\right\}^{-}$and $\Gamma_{0}\left(D_{A_{0}} S_{1} k \oplus\right.$ $\left.D_{S_{1}} k\right)=D_{S_{1}^{\prime}} A_{0} k, k \in \mathcal{K}_{0}$. Then $B$ satisfies $B U_{1}=U_{1}^{\prime} B, B U_{0}=U_{0}^{\prime} B, B U_{0}^{*}$ $=U_{0}^{\prime *} B$ and $P_{\mathcal{K}_{0}^{\prime}} B=A_{0} P_{\mathcal{K}_{0}}$ (see [9] for details). Hence $P_{\mathcal{H}^{\prime}} B=B P_{\mathcal{H}}$ and since $\left[\mathcal{K},\left(U_{0}, U_{1}\right)\right]$ and $\left[\mathcal{K}^{\prime},\left(U_{0}^{\prime}, U_{1}^{\prime}\right)\right]$ are the $*$-regular isometric dilations for $T$ and $T^{\prime}$ respectively, $B$ is the desired operator. The proof is finished.

Corollary 6. Let $[\mathcal{H}, T]$ and $\left[\mathcal{H}^{\prime}, T^{\prime}\right]$ be two bicontractions which have *-regular (or regular) isometric dilations with the minimal unitary extensions $[\widetilde{\mathcal{K}}, \widetilde{U}]$ and $\left[\widetilde{\mathcal{K}}^{\prime}, \widetilde{U}^{\prime}\right]$ respectively. If $A$ is a contraction from $\mathcal{H}$ to $\mathcal{H}^{\prime}$ which satisfies $A T_{i}=T_{i}^{\prime} A(i=0,1)$ and $A T_{0}^{*}=T_{0}^{\prime *} A$, then there exists a contraction $\widetilde{A}$ from $\widetilde{\mathcal{K}}$ to $\widetilde{\mathcal{K}}^{\prime}$ such that $\widetilde{A} \widetilde{U}_{i}=\widetilde{U}_{i}^{\prime} \widetilde{A}(i=0,1)$ and $P_{\mathcal{H}^{\prime}} \widetilde{A} \mid \mathcal{H}=A$. 
Proof. Suppose that $T$ and $T^{\prime}$ have the *-regular isometric dilations $[\mathcal{K}, U]$ and $\left[\mathcal{K}^{\prime}, U^{\prime}\right]$ and let $[\widetilde{\mathcal{K}}, \widetilde{U}]$ and $\left[\widetilde{\mathcal{K}}^{\prime}, \widetilde{U}^{\prime}\right]$ be the minimal unitary extensions of $U$ and $U^{\prime}$ respectively. If $A$ is an intertwining contraction of $T$ and $T^{\prime}$ and $B$ is an intertwining contraction of $U$ and $U^{\prime}$ with $P_{\mathcal{H}^{\prime}} B=A P_{\mathcal{H}}$ given by Theorem 5 , then there exists (see $[12]$ ) a contraction $\widetilde{B}$ from $\widetilde{\mathcal{K}}$ into $\widetilde{\mathcal{K}}^{\prime}$ which intertwines $\widetilde{U}$ and $\widetilde{U}^{\prime}$, such that $\widetilde{B} \mid \mathcal{K}=B$. It results that $P_{\mathcal{H}^{\prime}} \widetilde{B} \mid \mathcal{H}=A$, whence $P_{\mathcal{H}} \widetilde{B}^{*} \mid \mathcal{H}^{\prime}=A^{*}$ and $\widetilde{B}^{*}$ intertwines $\widetilde{U}^{\prime *}$ and $\widetilde{U}^{*}$. Obviously, $\left[\widetilde{\mathcal{K}}, \widetilde{U}^{*}\right]$ and $\left[\widetilde{\mathcal{K}}^{\prime}, \widetilde{U}^{\prime *}\right]$ are the regular unitary dilations of $T^{*}$ and $T^{*}$ respectively.

THEOREM 7. Let $[\mathcal{H}, T]$ and $\left[\mathcal{H}^{\prime}, T^{\prime}\right]$ be two bicontractions having regular isometric dilations $[\mathcal{K}, U]$ and $\left[\mathcal{K}^{\prime}, U^{\prime}\right]$ respectively, such that $T_{1}^{*}$ or $T_{1}^{\prime}$ is an isometry. If $A$ is a contraction of $\mathcal{H}$ into $\mathcal{H}^{\prime}$ such that $A T_{i}=T_{i}^{\prime} A(i=0,1)$ and $A T_{0}^{*}=T_{0}^{* *} A$, then there exists a contraction $B$ from $\mathcal{K}$ to $\mathcal{K}^{\prime}$ with $B U_{i}=U_{i}^{\prime} B(i=0,1)$, and $P_{\mathcal{H}^{\prime}} B=A P_{\mathcal{H}}$.

Proof. Suppose that the bicontractions $T=\left(T_{0}, T_{1}\right)$ and $T^{\prime}=\left(T_{0}^{\prime}, T_{1}^{\prime}\right)$ have regular isometric dilations. Then $T^{*}=\left(T_{0}^{*}, T_{1}^{*}\right)$ has a *-regular isometric dilation and therefore if $\left[\mathcal{K}_{0 *}, S_{0 *}\right]$ is the minimal isometric dilation of $T_{0}^{*}$, then there is a contraction $S_{1 *}$ on $\mathcal{K}_{0 *}$ which doubly commutes with $S_{0 *}$, such that $P_{\mathcal{H}} S_{1 *}=T_{1}^{*} P_{\mathcal{H}}$. Let $\left[\widetilde{\mathcal{K}}_{0}, \widetilde{S}_{0}\right]$ be the minimal isometric dilation of the coisometry $S_{0 *}^{*}$ and let $\widetilde{S}_{1}$ be the *-extension of $S_{1 *}^{*}$ to $\widetilde{\mathcal{K}}_{0}$ which doubly commutes with $\widetilde{S}_{0}$. But $\widetilde{S}_{0}$ is a unitary operator on $\widetilde{\mathcal{K}}_{0}$ and $\left[\mathcal{K}_{0}, S_{0}\right]$ given by

$$
\mathcal{K}_{0}=\bigvee_{n \in \mathbb{Z}_{+}} \widetilde{S}_{0}^{n} \mathcal{H}, \quad S_{0}=\widetilde{S}_{0} \mid \mathcal{K}_{0},
$$

is the minimal isometric dilation of $T_{0}$. We have $S_{1 *}^{*} \mid \mathcal{H}=T_{1}$ and therefore $\widetilde{S}_{1} \mid \mathcal{H}=T_{1}$. Hence $\mathcal{K}_{0}$ is an invariant subspace for $\widetilde{S}_{1}$ and $S_{1}=\widetilde{S}_{1} \mid \mathcal{K}_{0}$ is a contraction on $\mathcal{K}_{0}$ which satisfies $S_{0} S_{1}=S_{1} S_{0}$ and $S_{1} \mid \mathcal{H}=T_{1}$.

Analogously, if $\left[\mathcal{K}_{0}^{\prime}, S_{0}^{\prime}\right]$ is the minimal isometric dilation of $T_{0}^{\prime}$, then there is a contraction $S_{1}^{\prime}$ on $\mathcal{K}_{0}^{\prime}$ which satisfies $S_{0}^{\prime} S_{1}^{\prime}=S_{1}^{\prime} S_{0}^{\prime}$ and $S_{1}^{\prime} \mid \mathcal{H}^{\prime}=T_{1}^{\prime}$.

Now let $A: \mathcal{H} \rightarrow \mathcal{H}^{\prime}$ be a contraction which intertwines $T_{1}$ and $T_{1}^{\prime}$ and doubly intertwines $T_{0}$ and $T_{0}^{\prime}$. As in the proof of Theorem 5 there is a contraction $A_{0}: \mathcal{K}_{0} \rightarrow \mathcal{K}_{0}^{\prime}$ which doubly intertwines $S_{0}$ and $S_{0}^{\prime}$, such that $A_{0} \mid \mathcal{H}=A$. Then for any sequence $\left\{h_{n}\right\} \in \mathcal{H}$ with finite support we have

$$
\begin{aligned}
A_{0} S_{1} \sum_{n} S_{0}^{n} h_{n} & =\sum_{n} S_{0}^{\prime n} A_{0} S_{1} h_{n}=\sum_{n} S_{0}^{\prime n} A T_{1} h_{n} \\
& =\sum_{n} S_{0}^{\prime n} T_{1}^{\prime} A h_{n}=\sum_{n} S_{0}^{\prime n} S_{1}^{\prime} A_{0} h_{n}=S_{1}^{\prime} A_{0} \sum_{n} S_{0}^{n} h_{n},
\end{aligned}
$$

therefore $A_{0} S_{1}=S_{1}^{\prime} A_{0}$. Let us remark that if $T_{1}^{*}$ or $T_{1}^{\prime}$ is an isometry, then so is $S_{1}^{*}$ (respectively $S_{1}^{\prime}$ ). In this case it is known (see [5]) that $A_{0}$ has a unique contractive intertwining lifting of the minimal isometric dilations 
of $S_{1}$ and $S_{1}^{\prime}$. Now as in the proof of Theorem 5 (see [9]) we can obtain a contraction $B: \mathcal{K} \rightarrow \mathcal{K}^{\prime}$, where $\left[\mathcal{K}, U_{1}\right]$ and $\left[\mathcal{K}^{\prime}, U_{1}^{\prime}\right]$ are the minimal isometric dilations of $S_{1}$ and $S_{1}^{\prime}$ respectively, such that $P_{\mathcal{K}_{0}^{\prime}} B=A_{0} P_{\mathcal{K}_{0}}$ and $B U_{1}=U_{1}^{\prime} B, B U_{0}=U_{0}^{\prime} B, U_{0}$ and $U_{0}^{\prime}$ being the isometric extensions of $S_{0}$ and $S_{0}^{\prime}$ to $\mathcal{K}$ and $\mathcal{K}^{\prime}$ which commute with $U_{1}$ and $U_{1}^{\prime}$ respectively. Finally, it is easy to see that $\left[\mathcal{K},\left(U_{0}, U_{1}\right)\right]$ and $\left[\mathcal{K}^{\prime},\left(U_{0}^{\prime}, U_{1}^{\prime}\right)\right]$ are the regular isometric dilations of $T$ and $T^{\prime}$ respectively. The proof is finished.

Now we can obtain the versions of Theorems 5 and 7 for double intertwinings which complete those obtained in [14].

Proposition 8. Let $[\mathcal{H}, T]$ and $\left[\mathcal{H}^{\prime}, T^{\prime}\right]$ be two bicontractions having regular (or *-regular) isometric dilations $[\mathcal{K}, V]$ and $\left[\mathcal{K}^{\prime}, V^{\prime}\right]$ respectively. If $A$ is a contraction from $\mathcal{H}$ into $\mathcal{H}^{\prime}$ which doubly intertwines $T$ and $T^{\prime}$, then there exists a (unique) *-extension of $A$ from $\mathcal{K}$ to $\mathcal{K}^{\prime}$ which preserves the norm of $A$ and doubly intertwines $V$ and $V^{\prime}$.

P r o of. Suppose first that $T$ and $T^{\prime}$ have *-regular isometric dilations. Let $\left[\mathcal{K}_{0},\left(S_{0}, S_{1}\right)\right]$ and $\left[\mathcal{K}_{0}^{\prime},\left(S_{0}^{\prime}, S_{1}^{\prime}\right)\right]$ be as in the proof the Theorem 5. Consider $A: \mathcal{H} \rightarrow \mathcal{H}^{\prime}$ a contractive double intertwining of $T$ and $T^{\prime}$ and $A_{0}: \mathcal{K}_{0} \rightarrow \mathcal{K}_{0}^{\prime}$ with $A_{0} S_{i}=S_{i}^{\prime} A_{0}(i=0,1), A_{0} S_{0}^{*}=S_{0}^{\prime *} A_{0}, A_{0} \mid \mathcal{H}=A$, $A_{0}^{*} \mid \mathcal{H}^{\prime}=A^{*}$ and $\left\|A_{0}\right\|=\|A\|$. Then for $\left\{h_{p}\right\}_{p \geq 0} \subset \mathcal{H}$ with finite support, we have

$$
\begin{aligned}
A_{0} S_{1}^{*} \sum_{p} S_{0}^{p} h_{p} & =\sum_{p} S_{0}^{\prime p} A_{0} S_{1}^{*} h_{p}=\sum_{p} S_{0}^{\prime p} A T_{1}^{*} h_{p}=\sum_{p} S_{0}^{\prime p} T_{1}^{\prime *} A h_{p} \\
& =\sum_{p} S_{0}^{\prime p} S_{1}^{* *} A_{0} h_{p}=S_{1}^{*} A_{0} \sum_{p} S_{0}^{p} h_{p}
\end{aligned}
$$

and so $A_{0} S_{1}^{*}=S_{1}^{\prime *} A_{0}$.

Now let $\left[\mathcal{K}, V_{1}\right]$ and $\left[\mathcal{K}^{\prime}, V_{1}^{\prime}\right]$ be the minimal isometric dilations of $S_{1}$ and $S_{1}^{\prime}$ and let $V_{0}, V_{0}^{\prime}$ be the extensions of $S_{0}, S_{0}^{\prime}$ to $\mathcal{K}, \mathcal{K}^{\prime}$ which doubly commute with $V_{1}, V_{1}^{\prime}$ respectively. As above, there exists a contraction $B: \mathcal{K} \rightarrow \mathcal{K}^{\prime}$ with $B V_{i}=V_{i}^{\prime} B, B V_{i}^{*}=V_{i}^{*} B,(i=0,1), B\left|\mathcal{K}_{0}=A_{0}, B^{*}\right| \mathcal{K}_{0}^{\prime}=A_{0}^{*}$, whence $B\left|\mathcal{H}=A, B^{*}\right| \mathcal{H}^{\prime}=A^{*}$ and $\|B\|=\left\|A_{0}\right\|=\|A\|$. So the conclusion holds for the $*$-regular isometric dilations $[\mathcal{K}, V]$ and $\left[\mathcal{K}^{\prime}, V^{\prime}\right]$ of $T$ and $T^{\prime}$.

Next let $[\widetilde{\mathcal{K}}, \widetilde{U}],\left[\widetilde{\mathcal{K}}^{\prime}, \widetilde{U}^{\prime}\right]$ be the minimal unitary extensions of $V, V^{\prime}$ and $\left[\mathcal{K}_{*}, V_{*}\right],\left[\mathcal{K}_{*}^{\prime}, V_{*}^{\prime}\right]$ be the regular isometric dilations of $T^{*}, T^{*}$ respectively (as in (5)). Then there exists $([12],[7])$ a contraction $\widetilde{A}: \widetilde{\mathcal{K}} \rightarrow \widetilde{\mathcal{K}}^{\prime}$ such that $\widetilde{A} \widetilde{U}_{i}=\widetilde{U}_{i}^{\prime} \widetilde{A}(i=0,1), \widetilde{A}\left|\mathcal{K}=B, \widetilde{A}^{*}\right| \mathcal{K}^{\prime}=B^{*}$ and $\|\widetilde{A}\|=\|B\|$. Because $\widetilde{A} \mid \mathcal{H}=A$ and $\widetilde{A} \widetilde{U}_{i}^{*}=\widetilde{U}_{i}^{*} \widetilde{A}(i=0,1)$, we have $\widetilde{A} \mathcal{K}_{*} \subset \mathcal{K}_{*}^{\prime}$. But $\widetilde{A}^{*} \mid \mathcal{H}^{\prime}=A^{*}$ and $\widetilde{A}^{*} \widetilde{U}_{i}^{*}=\widetilde{U}_{i}^{*} A^{*}(i=0,1)$ imply $\widetilde{A}^{*} \mathcal{K}_{*}^{\prime} \subset \mathcal{K}_{*}$. So we can define the operator $C: \mathcal{K}_{*} \rightarrow \mathcal{K}_{*}^{\prime}$ by $C=\widetilde{A} \mid \mathcal{K}_{*}$. Then $C^{*}=\widetilde{A}^{*} \mid \mathcal{K}_{*}^{\prime}$ and $C \mid \mathcal{H}=A$, $C^{*} \mid \mathcal{H}^{\prime}=A^{*}$ and since $V_{* i}=\widetilde{U}_{i}^{*}\left|\mathcal{K}_{*}, V_{* i}^{\prime}=\widetilde{U}_{i}^{\prime *}\right| \mathcal{K}_{*}^{\prime}(i=0,1)$, it results that 
$C V_{* i}=V_{* i}^{\prime} C$ and $C V_{* i}^{*}=V_{* i}^{\prime *} C(i=0,1)$. Finally, $\|A\| \leq\|C\| \leq\|\widetilde{A}\|=$ $\|B\|=\|A\|$ and so $\|C\|=\|A\|$. Thus the conclusion holds for the regular isometric dilations of $T^{*}$ and $T^{* *}$, and consequently, in the case when $T$ and $T^{\prime}$ have regular isometric dilations.

Under certain conditions we can drop the doubly intertwining property on a component. The first fact in this context is contained in

Proposition 9. Let $[\mathcal{H}, T]$ be a bicontraction with the first component $T_{0}$ a coisometry, and $\left[\mathcal{H}^{\prime}, T^{\prime}\right]$ be another bicontraction which has a *-regular isometric dilation. Let $[\mathcal{K}, U]$ and $\left[\mathcal{K}^{\prime}, U^{\prime}\right]$ be the $*$-regular isometric dilations of $T$ and $T^{\prime}$ respectively. If $A$ is a contraction from $\mathcal{H}$ to $\mathcal{H}^{\prime}$ with $A T_{i}=T_{i}^{\prime} A$ $(i=0,1)$, then there exists a contraction $B$ from $\mathcal{K}$ to $\mathcal{K}^{\prime}$ such that $B U_{i}=$ $U_{i}^{\prime} B(i=0,1)$, and $P_{\mathcal{H}^{\prime}} B=A P_{\mathcal{H}}$.

Proof. Let $A: \mathcal{H} \rightarrow \mathcal{H}^{\prime}$ be a contractive intertwining of $T$ and $T^{\prime}$. Preserving the notations of the proof of Theorem 5, we find (by the lifting theorem) that there exists a contraction $A_{0}$ from $\mathcal{K}_{0}$ to $\mathcal{K}_{0}^{\prime}$ which satisfies $A_{0} S_{0}=S_{0}^{\prime} A_{0}$ and $P_{\mathcal{H}^{\prime}} A_{0}=A P_{\mathcal{H}}$. Because $T_{0}$ is a coisometry, its minimal isometric dilation $S_{0}$ is a unitary operator on $\mathcal{K}_{0}$. Then from Theorem B of [6] (which can be extended to operators acting on different spaces) it results that $A_{0} S_{0}^{*}=S_{0}^{\prime *} A_{0}$. So $A_{0}$ doubly intertwines $S_{0}$ and $S_{0}^{\prime}$. Furthermore, $A_{0}$ intertwines $S_{1}$ and $S_{1}^{\prime}$, the doubly commuting commutants of $S_{0}$ and $S_{0}^{\prime}$ which lift $T_{1}$ and $T_{1}^{\prime}$ respectively, given by Theorem 1 (iii). By Theorem 5 , $A_{0}$ has a contractive lift, which intertwines the $*$-regular isometric dilations of $S=\left(S_{0}, S_{1}\right)$ (of $T$ ) and $S^{\prime}=\left(S_{0}^{\prime}, S_{1}^{\prime}\right)$ (of $T^{\prime}$ ), whence the conclusion follows.

The dual version of Proposition 9 is in fact an extension of Proposition 5.2 from [12] (for bicontractions) and of Proposition 10 from [2].

Corollary 10. Let $[\mathcal{H}, T]$ be a bicontraction which has a regular isometric dilation, and $\left[\mathcal{H}^{\prime}, T^{\prime}\right]$ be a bicontraction with $T_{0}^{\prime}$ an isometry. We also suppose that $T_{1}^{*}$ or $T_{1}^{\prime}$ is an isometry. If $A$ is a contraction of $\mathcal{H}$ into $\mathcal{H}^{\prime}$ which intertwines $T$ and $T^{\prime}$, then there exists a contraction $B$ which intertwines the regular isometric dilations of $T$ and $T^{\prime}$ and satisfies $P_{\mathcal{H}^{\prime}} B=A P_{\mathcal{H}}$.

Proof. If $\left[\mathcal{K}_{0}, S_{0}\right]$ is the minimal isometric dilation of $T_{0}$ and $S_{1}$ is a contraction on $\mathcal{K}_{0}$ with $S_{0} S_{1}=S_{1} S_{0}$ and $S_{1} \mid \mathcal{H}=T_{1}$, then $A_{0}=A P_{\mathcal{H}}$ is a contraction from $\mathcal{K}_{0}$ into $\mathcal{H}^{\prime}$ and satisfies $A_{0} S_{0}=T_{0}^{\prime} A_{0}, A_{0} S_{1}=T_{1}^{\prime} A_{0}$, and $A_{0}$ is a lifting for $A$. Since $S_{0}$ and $T_{0}^{\prime}$ and respectively $S_{1}^{*}$ or $T_{1}^{\prime}$ are isometries, there is a lifting for $A_{0}$ which intertwines the regular isometric dilations for $\left(S_{0}, S_{1}\right)$ and $\left(T_{0}^{\prime}, T_{1}^{\prime}\right)$.

Recall ([13]) that a bounded linear operator $S$ on $\mathcal{H}$ is subnormal if there exists a normal operator $N$ on a Hilbert space $\mathcal{K} \supset \mathcal{H}$ such that $\mathcal{H}$ is 
invariant for $N$ and $S=N \mid \mathcal{H}$. If, furthermore, $\mathcal{K}=\bigvee_{p \geq 0} N^{* p} \mathcal{H}$, then $N$ is said to be the minimal normal extension of $S$. In this case, $N$ is unique (up to unitary equivalence) and $\|N\|=\|S\|$.

A bicontraction $T=\left(T_{0}, T_{1}\right)$ will be called semi-subnormal if one of the contractions is subnormal and the other one has an extension which commutes (therefore doubly commutes) with the minimal normal extension of the subnormal one. Such a bicontraction $T$ has a regular isometric dilation because, if $T_{0}$ is subnormal and $N_{1}$ is an extension of $T_{1}$ commuting with the minimal normal extension $N_{0}$ of $T_{0}$, then we have $\Delta_{T}=P_{\mathcal{H}} \Delta_{\left(N_{0}, N_{1}\right)} \mid \mathcal{H} \geq 0$.

It is easy to see that every subnormal bicontraction is semi-subnormal.

Now we have the following completion of Corollary 10.

Proposition 11. Let $T=\left(T_{0}, T_{1}\right)$ and $T^{\prime}=\left(T_{0}^{\prime}, T_{1}^{\prime}\right)$ be two semisubnormal bicontractions on $\mathcal{H}$ and $\mathcal{H}^{\prime}$ respectively, such that $T_{0}$ and $T_{0}^{\prime}$ are subnormal and $T_{1}^{\prime}$ is an isometry. If $A$ is a contraction from $\mathcal{H}$ to $\mathcal{H}^{\prime}$ which intertwines $T$ and $T^{\prime}$ and $A$ has an extension which intertwines the minimal normal extensions of $T_{0}$ and $T_{0}^{\prime}$, then $A$ has an extension which intertwines the regular isometric dilations of $T$ and $T^{\prime}$.

Proof. Let $T, T^{\prime}$ and $A$ be as in the hypothesis and let $[\widetilde{\mathcal{H}}, N]$ and $\left[\widetilde{\mathcal{H}}^{\prime}, N^{\prime}\right]$, where $N=\left(N_{0}, N_{1}\right)$ and $N^{\prime}=\left(N_{0}^{\prime}, N_{1}^{\prime}\right)$, be such that $N_{0}$ (resp. $N_{0}^{\prime}$ ) is the minimal normal extension on $\widetilde{\mathcal{H}}$ (resp. $\widetilde{\mathcal{H}}^{\prime}$ ) of $T_{0}$ (resp. $\left.T_{0}^{\prime}\right)$ and $N_{1}$ (resp. $N_{1}^{\prime}$ ) is a contraction on $\widetilde{\mathcal{H}}$ (resp. $\widetilde{\mathcal{H}}^{\prime}$ ) which extends $T_{1}$ (resp. $T_{1}^{\prime}$ ) and doubly commutes with $N_{0}$ (resp. $N_{0}^{\prime}$ ). From the hypothesis and the Fuglede-Putnam Theorem, there is an operator $\widetilde{A}: \widetilde{\mathcal{H}} \rightarrow \widetilde{\mathcal{H}}^{\prime}$ which doubly intertwines $N_{0}$ and $N_{0}^{\prime}$, and $\widetilde{A} \mid \mathcal{H}=A$. Then for $q \geq 0$ and $h \in \mathcal{H}$ we have

$$
\begin{aligned}
\widetilde{A} N_{1} N_{0}^{* q} h & =* \widetilde{A} N_{0}^{* q} N_{1} h=N_{0}^{\prime * q} \widetilde{A} T_{1} h=N_{0}^{\prime * q} A T_{1} h \\
& =N_{0}^{\prime * q} T_{1}^{\prime} A h=N_{0}^{\prime * q} N_{1}^{\prime} \widetilde{A} h=N_{1}^{\prime} \widetilde{A} N_{0}^{* q} h .
\end{aligned}
$$

Using the structure of the space $\widetilde{\mathcal{H}}$, it results that $\widetilde{A} N_{1}=N_{1}^{\prime} \widetilde{A}$. Let us remark that because $T_{1}^{\prime}$ is an isometry on $\mathcal{H}^{\prime}, N_{1}^{\prime}$ is also an isometry on $\widetilde{\mathcal{H}}^{\prime}$, hence the minimal unitary extension $\left[\mathcal{K}^{\prime}, V_{1}^{\prime}\right]$ of $N_{1}^{\prime}$ is just the minimal coisometry extension of $N_{1}^{\prime}$. Let $\left[\mathcal{K}, V_{1}\right]$ be the minimal coisometry extension of $N_{1}$ and let $V_{0}, V_{0}^{\prime}$ be the $*$-extensions of $N_{0}, N_{0}^{\prime}$ which doubly commute with $V_{1}, V_{1}^{\prime}$ respectively. Since $\widetilde{A}$ intertwines $N_{1}$ and $N_{1}^{\prime}$, there exists a contraction $\widetilde{A}_{1}$ from $\mathcal{K}$ into $\mathcal{K}^{\prime}$ which satisfies $\widetilde{A}_{1} V_{1}=V_{1}^{\prime} \widetilde{A}_{1}$ and $\widetilde{A_{1}} \mid \widetilde{\mathcal{H}}=\widetilde{A}$. In fact, $\widetilde{A}_{1}$ doubly intertwines $V_{1}$ and $V_{1}^{\prime}$ (see [6]). Moreover, $\widetilde{A}_{1}$ doubly intertwines the normal operators $V_{0}$ and $V_{0}^{\prime}$, because for $\left\{h_{n}\right\}_{n \geq 0} \subset \widetilde{\mathcal{H}}$ with finite support we have 


$$
\begin{aligned}
\widetilde{A}_{1} V_{0} \sum_{n} V_{1}^{* n} h_{n} & =\sum_{n} V_{1}^{\prime * n} \widetilde{A}_{1} V_{0} h_{n}=\sum_{n} V_{1}^{\prime * n} \widetilde{A} N_{0} h_{n} \\
& =\sum_{n} V_{1}^{\prime * n} N_{0}^{\prime} \widetilde{A} h_{n}=\sum_{n} V_{1}^{\prime * n} V_{0}^{\prime} \widetilde{A}_{1} h_{n}=V_{0}^{\prime} \widetilde{A}_{1} \sum_{n} V_{1}^{* n} h_{n} .
\end{aligned}
$$

Hence $\widetilde{A}_{1}$ doubly intertwines the bicontractions $V=\left(V_{0}, V_{1}\right)$ and $V^{\prime}=$ $\left(V_{0}^{\prime}, V_{1}^{\prime}\right)$. Then by Proposition $8, \widetilde{A}_{1}$ has an $*$-extension $\widetilde{B}$ which intertwines the regular isometric dilations $M=\left(M_{0}, M_{1}\right)$ on $\mathcal{M}$ of $V$ and $M^{\prime}=\left(M_{0}^{\prime}, M_{1}^{\prime}\right)$ on $\mathcal{M}^{\prime}$ of $V^{\prime}$ respectively. Setting

$$
\mathcal{K}=\bigvee_{n \in \mathbb{Z}_{+}^{2}} M^{n} \mathcal{H}, \quad \mathcal{K}^{\prime}=\bigvee_{n \in \mathbb{Z}_{+}^{2}} M^{\prime n} \mathcal{H}^{\prime}
$$

and $U_{i}=M_{i}\left|\mathcal{K}, U_{i}^{\prime}=M_{i}^{\prime}\right| \mathcal{K}^{\prime}(i=0,1)$, we deduce that $\left[\mathcal{K},\left(U_{0}, U_{1}\right)\right]$ and $\left[\mathcal{K}^{\prime},\left(U_{0}^{\prime}, U_{1}^{\prime}\right)\right]$ are the regular isometric dilations of $T$ and $T^{\prime}$ respectively. Since $\widetilde{B} \mathcal{H}=\widetilde{A} \mathcal{H} \subset \mathcal{H}^{\prime}$ and $\widetilde{B}$ intertwines $M_{i}$ and $M_{i}^{\prime}(i=0,1)$, it results that $\widetilde{B} \mathcal{K} \subset \mathcal{K}^{\prime}$. Consequently, $B=\widetilde{B} \mid \mathcal{K}$ is a contraction of $\mathcal{K}$ in $\mathcal{K}^{\prime}$ with $B U_{i}=U_{i}^{\prime} B(i=0,1)$ and $B \mid \mathcal{H}=A$.

Proposition 11 can be applied, in particular, to the case when $T$ is a subnormal bicontraction, which includes the spherical isometries ([2]) and the bidisc isometries ([4]).

Now we can give sufficient conditions for three commuting contractions in order to have unitary dilations.

THEOREM 12. Let $T_{0}, T_{1}, T_{2}$ be three pairwise commuting contractions on $\mathcal{H}$. If the bicontractions $\left(T_{0}, T_{1}\right)$ and $\left(T_{0}, T_{2}\right)$ have regular (or $*$-regular) isometric dilations, then $\left(T_{0}, T_{1}, T_{2}\right)$ has a isometric (unitary) dilation.

Proof. Suppose first that $\left(T_{0}, T_{1}\right)$ and $\left(T_{0}, T_{2}\right)$ have $*$-regular isometric dilations. Let $\left[\mathcal{K}_{0}, S_{0}\right]$ be the minimal isometric dilation of $T_{0}$ and $S_{1}, S_{2}$ be contractions on $\mathcal{K}_{0}$ which doubly commute with $S_{0}$, such that $P_{\mathcal{H}} S_{i}=T_{i} P_{\mathcal{H}}$, $i=1,2$. Then it results that $S_{1}^{*} S_{2}^{*}=S_{2}^{*} S_{1}^{*}$, and consequently, $S_{1} S_{2}=S_{2} S_{1}$ on $\mathcal{K}_{0}$. Let $\left[\mathcal{K}_{1},\left(V_{0}, V_{1}\right)\right]$ be the *-regular isometric dilation of $\left(S_{0}, S_{1}\right)$, therefore with $V_{0}$ and $V_{1}$ doubly commuting isometries on $\mathcal{K}_{1}$. By Theorem 5 there exists a contraction $V_{2}: \mathcal{K}_{1} \rightarrow \mathcal{K}_{1}$ with $P_{\mathcal{K}_{0}} V_{2}=S_{2} P_{\mathcal{K}_{0}}$ and $V_{2} V_{i}=$ $V_{i} V_{2}(i=0,1), V_{2} V_{0}^{*}=V_{0}^{*} V_{2}$. Therefore $V_{0}$ doubly commutes with $\left(V_{1}, V_{2}\right)$ and by Propositions 8 or 11, if $\left[\mathcal{K},\left(U_{1}, U_{2}\right)\right]$ is the regular isometric dilation of $\left(V_{1}, V_{2}\right)$, then there is an isometry $U_{0}$ on $\mathcal{K}$ such that $U_{0} \mid \mathcal{K}_{1}=V_{0}$ and $U_{0}$ commutes with $U_{1}$ and respectively with $U_{2}$. So for $m, n, j \in \mathbb{Z}_{+}$and $h \in \mathcal{H}$ we have

$$
\begin{aligned}
P_{\mathcal{H}} U_{0}^{m} U_{1}^{n} U_{2}^{j} h & =P_{\mathcal{K}_{1}, \mathcal{H}} P_{\mathcal{K}_{1}} U_{1}^{n} U_{2}^{j} V_{0}^{m} h \\
& =P_{\mathcal{K}_{1}, \mathcal{H}} V_{1}^{n} V_{2}^{j} V_{0}^{m} h=P_{\mathcal{K}_{0}, \mathcal{H}} P_{\mathcal{K}_{0}} V_{2}^{j} V_{0}^{m} V_{1}^{n} h
\end{aligned}
$$




$$
\begin{aligned}
& =P_{\mathcal{K}_{0}, \mathcal{H}} S_{2}^{j} P_{\mathcal{K}_{0}} V_{0}^{m} V_{1}^{n} h=P_{\mathcal{H}} S_{0}^{m} S_{1}^{n} S_{2}^{j} h \\
& =T_{0}^{m} P_{\mathcal{H}} S_{1}^{n} S_{2}^{j} h=T_{0}^{m} T_{1}^{n} P_{\mathcal{H}} S_{2}^{j} h=T_{0}^{m} T_{1}^{n} T_{2}^{j} h .
\end{aligned}
$$

Therefore $\left[\mathcal{K},\left(U_{0}, U_{1}, U_{2}\right)\right]$ is an isometric dilation of $\left(T_{0}, T_{1}, T_{2}\right)$.

Now if $\left(T_{0}, T_{1}\right)$ and $\left(T_{0}, T_{2}\right)$ have regular isometric dilations, then $\left(T_{0}^{*}, T_{1}^{*}\right)$ and $\left(T_{0}^{*}, T_{2}^{*}\right)$ have $*$-regular isometric dilations, so $\left(T_{0}^{*}, T_{1}^{*}, T_{2}^{*}\right)$ and consequently $\left(T_{0}, T_{1}, T_{2}\right)$ have isometric dilations.

Corollary 13. Let $T_{0}, T_{1}$ and $T_{2}$ be three pairwise commuting contractions on $\mathcal{H}$, such that $T_{0}$ is subnormal and $\left(T_{0}, T_{1}\right)$ and $\left(T_{0}, T_{2}\right)$ are semi-subnormal bicontractions. Then $\left(T_{0}, T_{1}, T_{2}\right)$ has a unitary dilation.

Proof. The bicontractions $\left(T_{0}, T_{1}\right)$ and $\left(T_{0}, T_{2}\right)$ have regular isometric dilations and we apply Theorem 12 .

Acknowledgements. The work of the first named author at this research is partially supported by the Alexander von Humboldt-Stiftung. The final form of the article was presented during his visit to the Universität des Saarlandes in Saarbrücken. The work of the second named author was partially completed during his visit to the University "La Sapienza" in Rome. We would like to thank Professors Ernst Albrecht and Stefano Marchiafava for their hospitality.

We are also very grateful to the reviewer for pointing out some misprints and language errors in the first variant of the paper.

\section{References}

[1] T. Ando, On a pair of commutative contractions, Acta Sci. Math. 24 (1963), 88-90.

[2] A. Athavale, On the intertwining of joint isometries, J. Operator Theory 23 (2) (1990), 339-350.

[3] S. Brehmer, Über vertauschbare Kontraktionen des Hilbertschen Raumes, Acta Sci. Math. (Szeged) 22 (1961), 106-111.

[4] R. E. Curto and F. H. Vasilescu, Standard operator models in the polydisc, Indiana Univ. Math. J. 42 (1993), 791-810.

[5] C. Foiass and A. E. Frazho, The Commutant Lifting Approach to Interpolation Problems, Birkhäuser, Basel, 1990.

[6] T. Furuta, An extension of the Fuglede-Putnam theorem to subnormal operators using a Hilbert-Schmidt norm inequality, Proc. Amer. Math. Soc. 81 (2) (1981), 240-242.

[7] D. Gaşpar and N. Suciu, Intertwining properties of isometric semigroups and Wold-type decompositions, in: Oper. Theory Adv. Appl. 24, Birkhäuser, Basel, 1987, 183-193.

[8] —, - On the Geometric Structure of Regular Dilations, Oper. Theory Adv. Appl., Birkhäuser, 1996.

[9] - - - On intertwining liftings of the distinguished dilations of bicontractions, to appear. 
[10] I. Halperin, Sz.-Nagy-Brehmer dilations, Acta Sci. Math. (Szeged) 23 (1962), 279-289.

[11] M. Kosiek, A. Octavio and M. Ptak, On the reflexivity of pairs of contractions, Proc. Amer. Math. Soc. 123 (1995), 1229-1236.

[12] W. Mlak, Intertwining operators, Studia Math. 43 (1972), 219-233.

[13] - Commutants of subnormal operators, Bull. Acad. Polon. Sci. Sér. Sci. Math. Astr. Phys. 19 (9) (1971), 837-842.

[14] V. Müller, Commutant lifting theorem for n-tuples of contractions, Acta Sci. Math. (Szeged) 59 (1994), 465-474.

[15] S. Parrott, Unitary dilations for commuting contractions, Pacific J. Math. 34 (1970), 481-490.

[16] M. Słociński, Isometric dilations of doubly commuting contractions and related models, Bull. Acad. Polon. Sci. Sér. Sci. Math. Astronom. Phys. 25 (12) (1977), $1233-1242$.

[17] —, On the Wold-type decomposition of a pair of commuting isometries, Ann. Polon. Math. 37 (1980), 255-262.

[18] B. Sz.- Nagy and C. Foiaş, Harmonic Analysis of Operators on Hilbert Space, North-Holland, Amsterdam-Budapest, 1970.

Department of Mathematics

University of Timişoara

Bv. V. Pârvan 4

1900 Timişoara, Romania

E-mail: gaspar@tim1.uvt.ro 